\title{
Ultrasonografía en niños con dolor agudo de la cadera
}

\author{
Cristián García B. ${ }^{1}$; María Elena Navarro 0. ${ }^{1}$; José Domingo Arce V. ${ }^{4}$; \\ Felipe Toro S $^{2}$; Eduardo Talesnik G. ${ }^{3}$
}

\section{Ultrasonography in children with acute hip pain}

\begin{abstract}
Ac.te hip pain is a frequent symplon in children and may be secondary to several causes, including transient synovitis, seplic arthritis, trauma, osleomyelitis, socroiliac arthritis. It is very importont to make a prompl ond precise diagnosis in order to select an adequate Ireatment and to avoid polenlial sequelae. Ultrasonography is a non irivasive ond isocuous imaging method, highly sensitive, even more thon X-ray films, for hip joinl effussion. Our experience in 27 children wilh ceute hip pain who were evalucled by ultrosonogrophy is described. Joiril effussion was found in 23 of these patients, whose tinal diagnosis was transient synovilis in sevenleen cases, septic arthritis in five ond hemarthrosis in one Utrasonography showed no obnormalities in three patients. Wwo had sacroitiac arlhritis and in one cose symploms were altributed to muscular distention. In a five day old girl with limited hip ioint motion, congenifal hip dysplasia was shown by ultrasonogrophic examinotion. In all our potients, ulliasonography was. esserilial for prompl diagnoss and management avoiding the use of other diagnostie imoging methods in most of them. We srlongly recommend this imaging technique in every children with acute hip pain
\end{abstract}

iKey words: hip joint, poin, diagnosis, ultrasonagraphy .)

El dolor agudo de la cadera es un motivo de consulta frecuente en la práctica pediátrica y constituye un sintoma relevante, dada la importancia de establecer un diagnóstico etiológico precoz y certero, para así determinar una conducta terapéutica apropiada en cada caso ${ }^{1-3}$. Puede ser secundario a sinovitis transitoria, artritis séptica, tarumatismo, osteomielitis, sacroileitis, tumores, entre otras afecciones. Es difícil diferenciar clínicamente estas distintas causas, debido a la localización profunda de la articulación de la cadera, que dificulta su examen clínico $y$, ademas, porque en las enfermedades de la cadera el dolor puede ser referido a otro segmento anatomico 4 .

Las radiografías $(\mathrm{Rx})$ simples de pelvis pueden no mostrar alteraciones en estos casos ${ }^{4}$. .

1. Departamenı de Radiología, Hospital Clínico Universidad Católica de Cbile.

2. Departamento de Traumatología. Hospital Clínico Universidad Católica de Chile.

3. Departamento de Reumatología, Hospital Clínico Universidad Católica de Chile.

4. Servicio de Radiología, Clínica Santa María.
La ultrasonografía (US) es un método sencillo, inocuo y de alta sensibilidad para detectar derrame articular de la cadera, 10 que ha resultado de gran utilidad en la evaluación de niños con dolor agudo de esta articulación', 2. 5.6.

En seguida se describe una experiencia con ultrasonografía en el diagnostico de $27 \mathrm{ni}$ nos que consultaron por dolor agudo de la cadera.

\section{Pacientes y Método}

Se estudiaron 27 niťos, 15 varones y 12 niñas, cuya edad fluctuaba entre 5 dias y 12 affos ( $\bar{x}=4,2$ anos), que consultaron por dolor agudo de cadera en los servicios de urgencia del Hospital Clínico de la Universidad Católica y de la Clínica Sanıa María, de Santiago. Todos ellos presentaban como síntoma fundamental dolor a limitación funcional de una cadera. Cinco terían fiebre al consultar, en 1S se registraban antecedentes de una enfermedad presuntamente viral previa y uno, portador de trastorno de la coagulación por déficit de factor VIJl, había sufrido un traumatismo leve de la cadera comprometida. En nueve niños se realizaron exámenes de laboratorio. incluyendo hemograma y velocidad de hemosedimentacion (VHS). En 25 pacientes se bicieron radiografias simples de pelvis, en 
proyecciones anteroposterior y en abducción y rotación externa (Lauenstein) para evaluar los espacios articulares de las caderas, buscar lesiones óseas y alteraciones de las partes blandas.

En todos los pacientes se efectuó ultrasonografía de anbas caderas con equipo de alta resolución (ALOKA 630 ) y tranductores de $7,5 \mathrm{MHz}$, en cortes sagitales obliLuos. En ningún caso este cxanen duró más de 7 minutos y sienpre se efectuó entre 20 y 90 minutos después de la consulta. El mérodo permite identificar, normalmente, el segmen1o proximal del fémur, incluyendo epifisis, metáfisis, diátísis, cápsula articular, músculo glúteo menor $y$ el cartílago. Este último aparece como una banda anecogéajca que rodea al hueso (figura l): en los casos normales no es visible el espacio anticular, por ser virtual.

En tres pacientes se hizo cintigtafio osen. En seis se efectuó punción articular de la cadera comprometida, con esıdudi cíłoquínuco y bacteriológico del líquido anticular. En tres pacientes con diagnóstico de sinovitis transitoria se efecttó ulirasonografŕa de control hasta 14 días despues de iniciada la enfermedad.

\section{Resultados}

Sólo en 15 de los 25 pacientes en quienes se obtuvieron radiografias de pelvis, se observaron alteraciones, las que consistieron fundamentalmente en aumento de Ia amplitud del espacio articular de la cadera afectada con respecto a la contralateral (figura 2). La ultrasonografía mostró hallazgos característicos de derrame articu- lar de cadera en 23 pacientes, con líquido intraarticular, distension, rechazo y engrosamiento de la cápsula articular (figura 3). De éstos, 17 correspondieron clínicamente a sinovitis transitoria y evolucionaron bien, con desaparición de los síntomas en un promedio de 7 días. Cinco sufrían artritis séptica, demostrada por punción articular, estudio citoquímico y bacteriológico del líquido articular en todos; en cuatro se registraban ecos en el líquido articular; todos fueron tratados quirúrgicamente, con drenaje abierto de la articulación, entre 45 y 90 minutos después de efectuado el diagnóstico. En el paciente con antecedentes de alteracion en la coagulación, la ultrasonografía mostró derrame articular de la cadera con múltiples ecos en su interior (figura 4). En una paciente de 5 días de edad, en quien se sospechaba clínicamente de artritis séptica de cadera, por mostrar limitación de la movilidad y dolor de la articulación, se encontró, mediante la ultrasonografía, que sufría de displasia de cadera. En 3 niños la ultrasonografía no mostró alteraciones y de éstos, 2 cortespondieron a sacroileitis, demostrada por cintigrafía osea. En un niño, en que el dolor desapareció espontáneamente en 48 horas, las molestias fueron atribuidas a distension muscular. En los tres pacientes con sinovitis transitoria en que se efec-

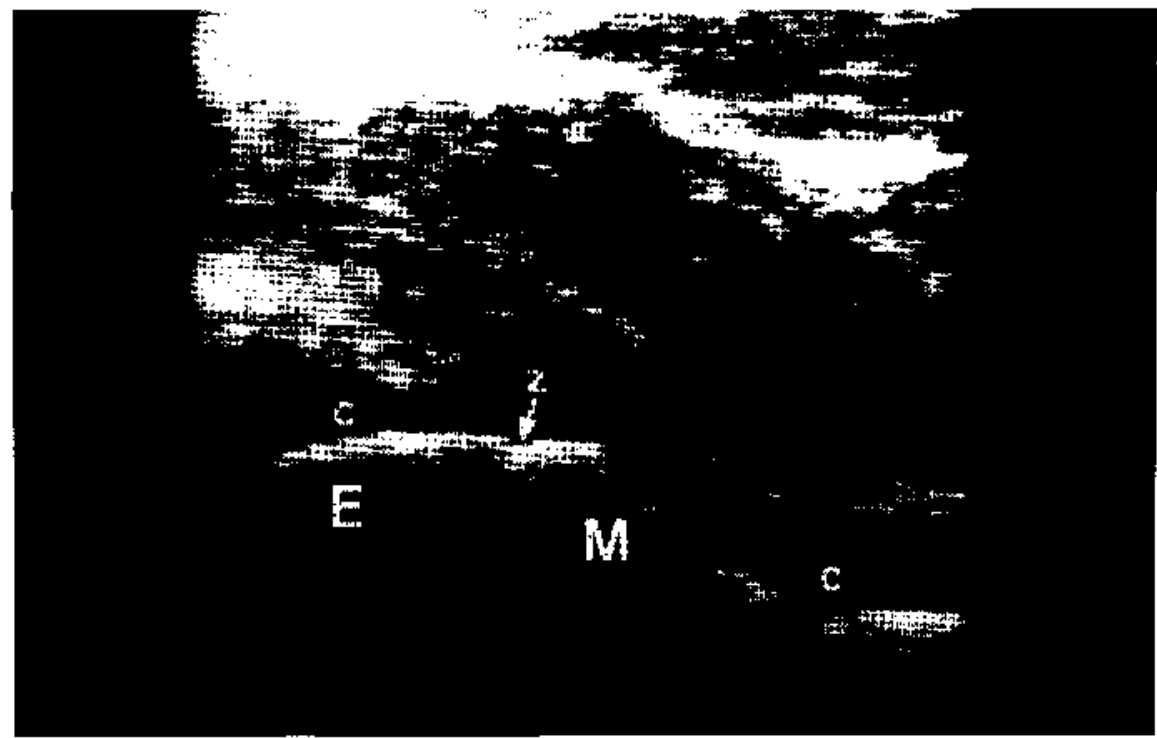

Figura 1: Aspecto normal de la ultrasonografia de cadera en corte sagital oblicuo. Son visibles el fémur. la metáfısis (M), la zoná de crecimiento (z) y la epífisis (E). La cápsula articular delimita el espacio articular (flechas). El cartílago óseo es de baja ecogenicidad (c). En la cara anterior se ve el músculo iliopsoas en su porción inferior (IP). 


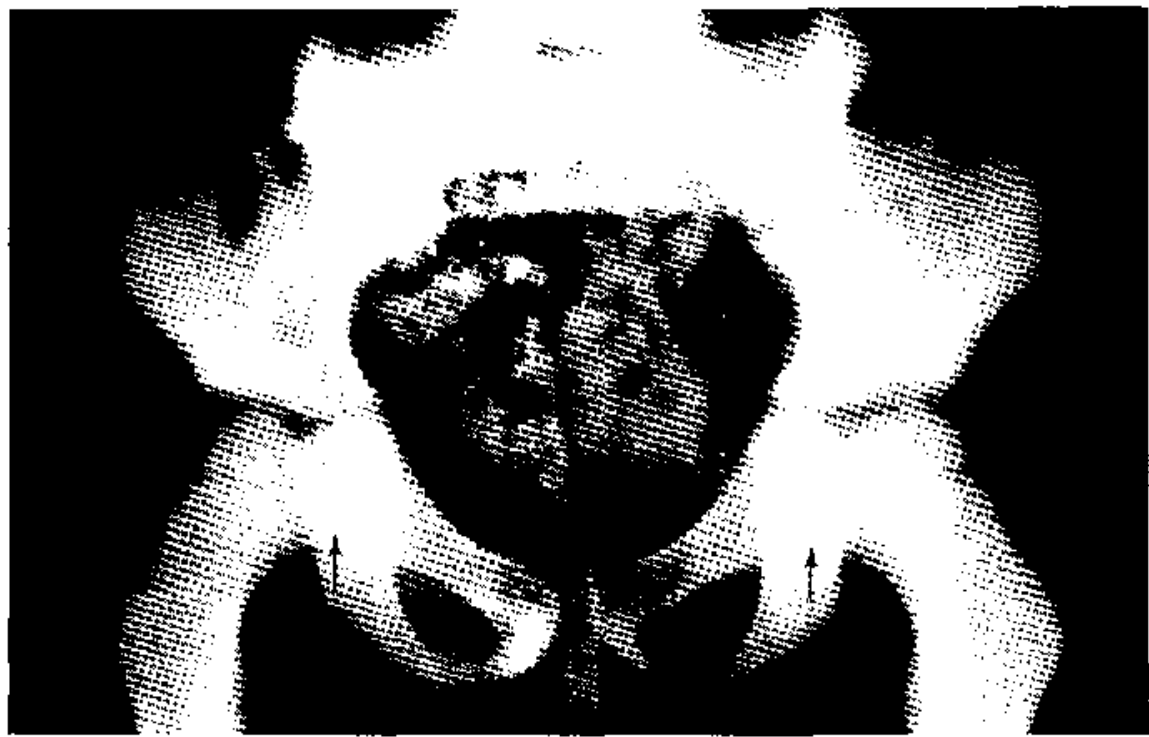

Figura 2: Nin̂o de 5 años con sinovitis transitoria de tadera derecha. La radiografía inuestra discyeto ensanthamiento del sspacio articular de la cadera derecha con respecto a la izquierda

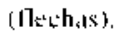
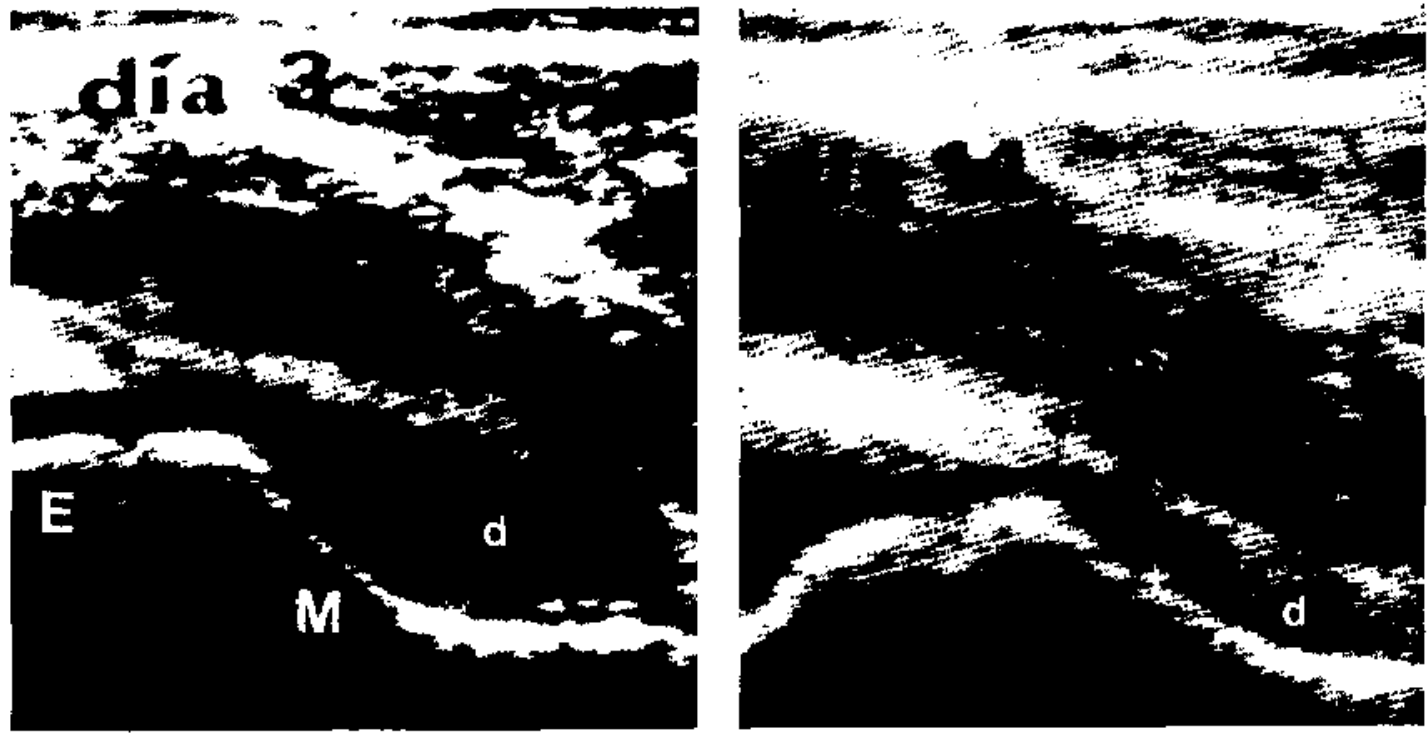

I igura 3: En el insmo niño. là vltiasonografía al tercer día de evolución (a) muesira delrame articular de: la cadera derechia (d) y desplazamento de la capsula articular (Пechas). Son visibles la metafisis (M) y la epífisis (E) del fémur. A los 14 dias (b) al derrome articulir (d) ha disnlinuido notablemente

tuci seguimiento con ultrasonografía, se observo disminución progresiva del derrame articular, cor persistencia de sólo pequeña cantidad dc liquido articular, 14 dias después de iniciadas las molestias.

\section{Comentario}

El dolor agudo de cadera es un motivo de consulta frecuente en niños y puede constituir un dilema diagnóstico en csia edad, ya que los 


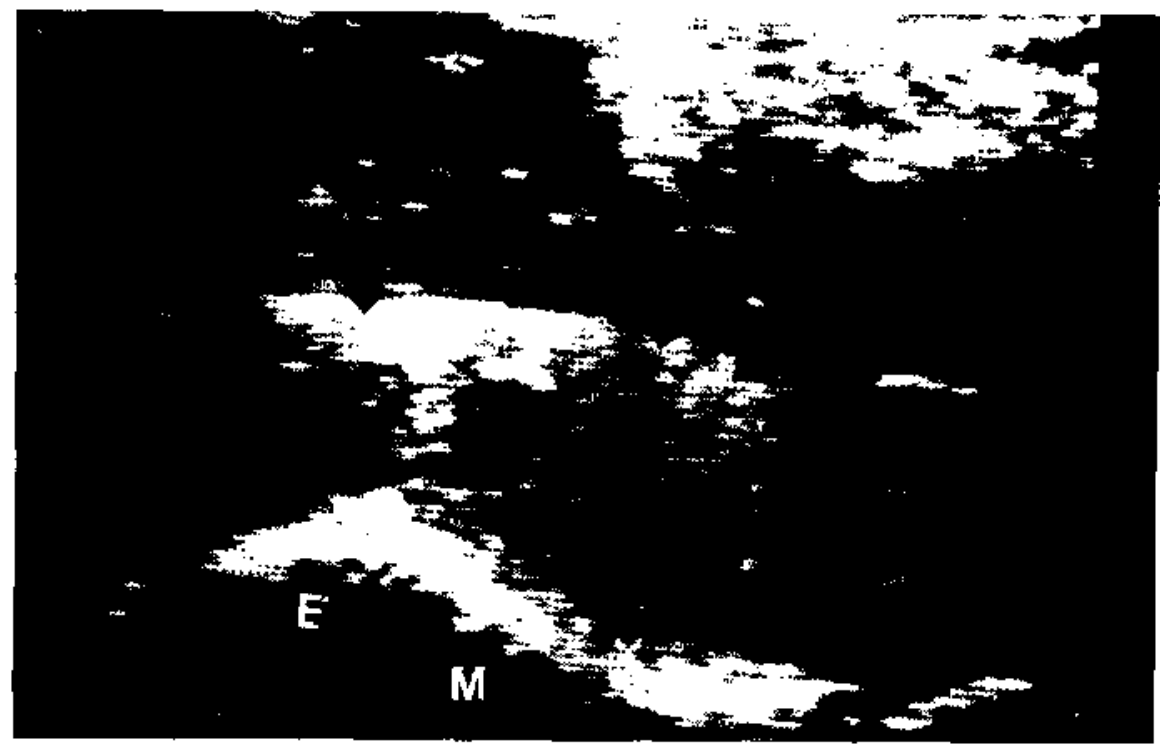

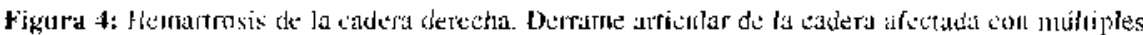
wos pequeinox en su interior (d). La cipsula articular (flechas) está abombada. M: nletátisis fiemaral y F: epifisis temoral

síntomas y hallazgos del examen físico pueden ser vagos c inespecíticos. Es fundamental establecer un diagnóstico cliológico preciso en estos pacientes. para así determinar una conducta terapéutica adceuada. Su causa más común es la sinovitis transitoria ${ }^{3.7}$, cuya eliología y patogenia no son bict conocidas, pero probablentente su causa es viral, considerando su evolución elínicit, el resultado de los exámenes de laboratorio y la alta frecuenciá de antecedentes de presunta infección viral previa ${ }^{?+3}$. En la mayoria de Jos casos la sinovitis ransitoria es unilateral, de pronóstico benigno y su tratamiento es médicos s. 7: ocurte con mayor frecuencia en niños

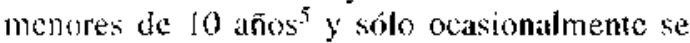
registlan antecedentes de pequeño traumatismo asociado ${ }^{? .}$; se manifiesta clínicamente por dohor e importencia funcionai de la cadera afectada. gue cede con reposo y analgésicos en un plazo no mayor a 7 días ${ }^{3.5}$. Clínicamente, el diagnóstice diferencial fundamental de la sinovitis transitoria es con la artitis séptica de cadera, que generalmente produce mayor impotencia funciomal. sucle acompañalse de fiebre y se asocia, además, a leucocilosis, desviación a la izquierda de la lormula leucowilaria, aumento de la velocidad de scedimentación globular (VHS) $)^{1.2}$. Esta atección tequiere tratamiento quirúrgico preco\%. con drenaje de la articulación, pará evitar complícaciones. como daño del cartilago epifisiario y osteomielitis. En la cadera puede tambien manifestarse dolor referido. como en la sacroiteiis ${ }^{8 .}{ }^{9}$, fenómeno observado en dos de nucstros pacientes.

La cadera es una articulación prolunda, difícil de examinar clínicannente. por lo que muchas veces se requieren métodos de diagnóstico por imágenes para su mejor evaluación. ales como las radingrafías simples. ultrasonogralías, cintigrafías óseas y eventualmente olros, como tomografías axiales computadorizadas o resonancia magnćtica.

Cuando hay derrame articular, la radingrafía de pelvis pucde ser normal o mostrar aumerto cn la amplitud del espacio arlicular y en el volumen de las partes blandas vecinas de la cadera afectada $^{2.6}$. En los casos de osteomielitis a sacruiletis. la radiografia puede mostrar lesiones óseas caraclerísticas, pero éstas generalmente aparceen en forma tardía. En cstos casos da cinligrafía es de mucho mayor utilidad y da resultados positivos desde aproximadamente 24 horas despućs de comenzada la enfermedad.

La ultrásonografía cs un mćtodo rápido, sencillo, inocuo, que no tequicre sedación ni empleo de radiación ionjzante, que ha mostrado 
gran sensibilidad para deteclar derrames articulares de la cadera ${ }^{1.2 .4 .6}$, registrando algunos tan pequeños como de 1 a $2 \mathrm{ml}^{1.8}$. Sin embargo, la detección de Jerrame articular, en general, no permite diferenciar con certeza entre sinovitis transitoria y artritis séptica y son más bien los elementos de juicio clínicos los que orientan sobre su causa. Sólo cuando se registran ecos en el interior del líquido articular se puede sospechar que se trata de artritis séptica ${ }^{4}$, lo que ocurrio en tres de los cinco pacientes con esa afección de esta serie. Ese fenómeno también puede observarse tn casos de hemartrosis. En todo caso la delección de derrame articular confirma que los síntomas provienen de la cadera. Por el contratio. cuando la ultrasonografía no muestra alteraciones, debe considerarse la posibilidad de un dolor referido a la cadera, fundamentalmente sactóleitis o. también, osteomielitis del fémur proximal, para cuya identificación la cintigrafía osca es el método ideal ${ }^{8}$. La resonancia magnética lienc también alto rendimiento en estos casos".

La ultrasonografía es más sensible que la radiografía en la detección de dertame articular de li caderal. 2.6. En las radiografías de pelvis, un aumento discreto de la amplitud del espacio arricular puede también. corresponder a una va. riante normal t.

La ultrasonografía pucde ser considerada el mêtodo de elección para detectar y scguir derrames arliculares de la cadera ${ }^{4}$ en niños y puede, además, servir de guía para realizar punción y alspiración con tĩnes de diagnóstico durante el mismo examen, especialmente en casos en que se sospecha artritis septica o cuando el derrame es pequeño, con lo que se ahorra tiempo y se pucden evitar punciones repetidas innecesarias ${ }^{2}$.

Recomendamos al uso de la ultrasonografía de cadera ел niños con dolor agudo de esta articulación, considerando que es un método no invasivo, rápido, de bajo costo y de alta sensibilidad que permite una buana aproximación al diagnóstico.

\section{Resumen}

El dolor agudo de cadera es un motivo frecucnte de consulta en pediatría. Puede ser se- cundario a distintas afecciones, incluyendo sinovitis transitoria, artritis séptica, traumatismo, osteomielitis y sacroileitis. Es importante establecer precozmente el diagnóstico preciso para definir la conducta terapéutica y evitar potenciales secuelas. La ulırasonografía es un método no invasivo, inocuo, de alta sensibilidad en la detección de derrames de esta articulación. En una serie de 27 niños con dolor agudo de la cadera, se encontró dertame articular en 23,17 de los cuales correspondicron a sinovitis transitoria, 5 a artritis séptica y uno a hemartrosis. El examen no mostró alteraciones en tres niños, dos de los cuales correspondieron a sacroileitis $y$ en uno, los síntonas fueron atribuidos a distensión muscular. En todos estos pacientes la ultrasonografía fue un elemento orientador fundamental en el diagnóstico etiológico del dolor de cadera y evitó efectuar exámenes más complicados, en la mayor parte de ellos. Recomendamos el uso de esta técnica en el estudio de todo niño con dolor agudo de cadera.

(Palabras clave: artritis, cadera, transitoria, séptica, sacroilíaca, ultrasonografía.)

\section{Referencias}

I. Zieger $M$, Durr U, Schulz R: Ultrasonography of hip joint effusions. Skeletal Radiol 1987; 16:607-6II.

2. Zawin $J$, Hoffer F. Rand F: Joint effusion in children with an irritable hip: US diagnosis and aspiration. Radiology 1993: 187: 459-463.

3. Spock A: Transient synovitis of the hip joinc in children. Pediatrics 1959: 24: 1042-1045.

4. Marchal GJ, Van Holbeech MT. Rates $M$. et al.: Transient Synovitis of the hip in children: role of US. Radiology 1987: 162: 825-828.

5. Peck R.I: Ultrasound of the painful hip in children, $\mathrm{Br}$ J Radiol 1986: 59: 29.3.294.

6. Adun R, Hendry GM. Moss $J$, et at.: Arthrosonography of the irritable hip in childhood: a review of 1 year's experience. Br J Radiol 1986; 59: 205-208.

7. Adkishs JA: Transient synovitis of the hip joint in children. J Bone Joint Surg (B) 1963: 45: 471-476.

8. Miller $J H$, Gates GF: Scintigraphy of sacroiliat pyarthrosis in children. JAMA 1977:238:2701-2704.

9. Halilogin $M$. Kleinman $M B$. Siddique $A L$. et al.: Osteomielitis and pyogenic infections of the sacroiliac joinl. MRI findings. Pediat Radiol 1994; 24: 3,33335.

10. Navarro ME. Garcia C. Arce $D$, el al.: Dolor agudo de cadera en el niño: utilidad del ultrasoujdo. Trabajo presentado en Jornadas Nacionales de Radiología. 1991. 